\title{
Doping-Induced Magnetization Plateaus
}

\author{
Holger Frahm and Constantin Sobiella \\ Institut für Theoretische Physik, Universität Hannover, D-30167 Hannover, Germany
}

(Received 6 July 1999)

\begin{abstract}
The low-temperature magnetization process of antiferromagnetic spin- $S$ chains doped with mobile spin- $(S-1 / 2)$ carriers is studied in an exactly solvable model. For sufficiently high magnetic fields the system is in a metallic phase with a finite gap for magnetic excitations. In this phase, which exists for a large range of carrier concentrations $x$, the zero-temperature magnetization is determined by $x$ alone. This leads to plateaus in the magnetization curve at a tunable fraction of the saturation magnetization. The critical behavior at the edges of these plateaus is studied in detail.
\end{abstract}

PACS numbers: 75.10.Jm, 71.10.Pm, 75.10.Lp

Synthetization of new magnetic materials and availability of very high magnetic fields provide new possibilities to study the magnetization process of low-dimensional quantum spin systems. In particular, so-called spin liquids realized in quasi-one dimensional antiferromagnetic systems such as spin chains, spin ladders, and exchangealternating spin chains, attract much interest at present due to the possible occurrence of magnetization plateaus associated with gapped excitations. In addition to saturated magnetization $M_{s}$, such plateaus, i.e., regions where the magnetization does not depend on the magnetic field for sufficiently low temperatures, are admissible from topological considerations at certain fractions of $M_{s}$ depending on the value of the spin of the substance and the translational symmetry of the ground state. Necessary conditions for the occurrence of plateaus have been formulated by Oshikawa et al. [1] employing a generalization of the Lieb-Schultz-Mattis theorem: for a spin- $S$ chain with a magnetic unit cell containing $q$ magnetic moments this feature can appear at rational values $\langle M\rangle$ with integer $q(S-\langle M\rangle)$. The existence of these phenomena in a variety of models has been established by numerical and analytical studies of various low-dimensional magnetic insulators including spin chains, spin ladders, and systems with multi spin exchange or exchange anisotropies [2-13]. Very recently, several experimental observations of such magnetization plateaus at nonzero $\langle M\rangle$ have been reported [14-16].

A common feature in these systems is that the plateaus in the magnetization curves appear at certain simple fractions of the maximal value $M_{s}$ as a consequence of their topological origin. In this Letter we report on a mechanism leading to gaps for magnetic excitations at magnetizations which can be controlled by suitable preparation of the sample, namely, doping. We study this phenomenon in the framework of a recently introduced class of integrable models for doped Heisenberg chains which may be used as a basis for studies of certain features of doped transition metal oxides $[17,18]$. Starting from the doubleexchange model [19], a strong ferromagnetic Hund's rule coupling between the spins of the itinerant $e_{g}$ electrons and localized quantum spins $(S-1 / 2)$ arising from the $t_{2 g}$ electrons allows ones to introduce an effective Hamiltonian on a restricted Hilbert space with maximally allowed spin $S^{\prime}$ on a given lattice site [20,21]; i.e., $S^{\prime}=S$ if the electronic state on this site is occupied, or $S^{\prime}=S-1 / 2$ if there is no $e_{g}$ electron (denoted as a hole in the following). This derivation of a low-energy Hamiltonian generalizes that of the $t-J$ model from the Hubbard model [22] which it corresponds to the case of $S=1 / 2$, i.e., no localized spins. Numerical studies of the $S \geq 1$ variants of these models have been performed to gain a better understanding of experimental findings for the doped Haldane system $\mathrm{Y}_{2-x} \mathrm{Ca}_{x} \mathrm{BaNiO}_{5}(S=1)$ [20] and manganese oxides such as $\mathrm{La}_{1-x} \mathrm{Ca}_{x} \mathrm{MnO}_{3}(S=2)$ [23].

Below we consider integrable models of this type in one spatial dimension. Similar to the models obtained from the general procedure outlined above, their Hamiltonians are of the form

$$
\mathcal{H}^{(S)}=\sum_{n=1}^{L}\left\{\chi_{n, n+1}^{(S)}+\mathcal{T}_{n, n+1}^{(S)}\right\} .
$$

Here $\chi_{i j}^{(S)}$ and $\mathcal{T}_{i j}^{(S)}$ describe the (antiferromagnetic) exchange and hopping of the holes between sites $i$ and $j$ of the lattice, respectively. SU(2) invariance of the model implies that $\chi^{(S)}\left(\mathcal{T}^{(S)}\right)$ can be written as polynomials of degree $2 S(2 S-1)$ of the operator $\mathbf{S}_{i} \cdot \mathbf{S}_{j}$ (see, e.g., [21]). The form of these polynomials is fixed in the integrable models [18]. For example, in the $S=1$ case with possible relevance to the doped nickel oxides, the antiferromagnetic exchange terms are given in terms of bilinear and biquadratic Heisenberg couplings depending on the values $S_{i, j} \in 1 / 2,1$ of the spins on sites $i$ and $j$ [17]:

$$
\begin{aligned}
\chi_{i j}^{(1)}=\frac{1}{2}( & \frac{1}{S_{i} S_{j}} \mathbf{S}_{i} \cdot \mathbf{S}_{j}-1 \\
& \left.+\delta_{S_{i} S_{j}, 1}\left[1-\left(\mathbf{S}_{i} \cdot \mathbf{S}_{j}\right)^{2}\right]\right) .
\end{aligned}
$$

(Note that the undoped chain, i.e., $S_{i}=1$ for all $i$, is the integrable spin-1 Takhtajan-Babujian model $[24,25]$ while the completely doped chain is the spin- $1 / 2$ Heisenberg chain with bilinear exchange.) Similarly, the kinetic term of the integrable spin- 1 model reads

$$
\mathcal{T}_{i j}^{(1)}=-\left(1-\delta_{S_{i}, S_{j}}\right) \mathcal{P}_{i j}\left(\mathbf{S}_{i} \cdot \mathbf{S}_{j}\right),
$$


where $\mathcal{P}_{i j}$ is an operator permuting the states on sites $i$ and $j$ thereby allowing the spin- $1 / 2$ "holes" to propagate. The additional exchange term in this expression leads to different hopping amplitudes $t\left(S_{i j}\right)$ depending on the total spin $S_{i j}$ on the participating sites, i.e., $t(1 / 2)=-1$ and $t(3 / 2)=+1 / 2$ for the possible values $S_{i j}=1 / 2$ and $3 / 2$, respectively. These amplitudes differ from the values proposed in Ref. [20] for the doped nickel oxide, namely, $t(1 / 2)=-1 / 2, t(3 / 2)=1$. This is one reason for the absence of a ferromagnetic phase in the integrable model (1) (see [17] for a discussion of the other differences).

For general $S$ the integrable models are constructed from solutions of a Yang-Baxter equation and can be solved by means of the algebraic Bethe ansatz [18]. Their thermodynamical properties at finite temperature $T$ can be obtained from the solution of the thermodynamic Bethe ansatz (TBA) equations, i.e., the following set of coupled nonlinear integral equations

$$
\begin{aligned}
\epsilon_{n}(\xi)= & T s * \ln \left[1+\mathrm{e}^{\epsilon_{n-1}(\xi) / T}\right]\left[1+\mathrm{e}^{\epsilon_{n+1}(\xi) / T}\right] \\
& -2 \pi \delta_{n, 2 S} s(\xi)-\delta_{n, 1} T s * \ln \left[1+\mathrm{e}^{-\kappa(\xi) / T}\right], \\
& -\left[2 \pi a_{2 S} * s(\xi)+\mu\right]-T s * \ln \left[1+\mathrm{e}^{\epsilon_{1}(\xi) / T}\right] \\
= & \kappa(\xi)+T R * \ln \left[1+\mathrm{e}^{-\kappa(\xi) / T}\right] .
\end{aligned}
$$

Here $(f * g)(\xi)$ denotes a convolution in the space of rapidities $\xi, \quad a_{n}(\xi)=(2 n / \pi)\left(4 \xi^{2}+n^{2}\right)^{-1}$, $s(\xi)=(2 \cosh \pi \xi)^{-1}, \quad$ and $\quad R=a_{2} *\left(1+a_{2}\right)^{-1}$. Equations (2) are to be solved subject to the condition $\lim _{n \rightarrow \infty}\left(\epsilon_{n} / n\right)=H$ with the external magnetic field $H$, and $\mu$ is the chemical potential for the holes controlling their concentration. In terms of the functions $\epsilon_{n}(\xi)$ and $\kappa(\xi)$, the free energy of this system reads as follows $\left(E_{0}^{(S)}\right.$ is the ground state energy of the spin- $S$ Takhtajan-Babujian chain for $H=0$ [25]):

$$
\begin{aligned}
\frac{1}{L} F(T, H, \mu)= & \frac{E_{0}^{(S)}}{L}-T \int \mathrm{d} \xi s(\xi) \ln \left[1+\mathrm{e}^{\epsilon_{2 S}(\xi) / T}\right] \\
& -T \int \mathrm{d} \xi\left(a_{2 S} * s\right)(\xi) \ln \left[1+\mathrm{e}^{-\kappa(\xi) / T}\right] .
\end{aligned}
$$

The low temperature $(H \gg T)$ phase diagram for the spin-1 system has been obtained in Ref. [17]; qualitatively the same behavior is found for general $S \geq 1$ [18].

Here we study the properties of these systems in a magnetic field at fixed doping. For hole concentrations $0<$ $x<x_{c}(S)$ (see Fig. 1) the low-energy excitation spectrum of the system allows one to identify four intermediate field phases (labeled $A, B_{1}, C$, and $B_{2}$ in Fig. 1 of Ref. [17]) for $0<H<H_{s}$ before the system is completely polarized for $H>H_{s}$. Of particular interest in the present context is the phase $C$ : since the system is not ferromagnetically polarized one expects nontrivial excitations for both the charge and magnetic degrees of freedom. In the neighboring phases $B_{1,2}$ these excitations are massless leading to an effective description of these phases in terms of a

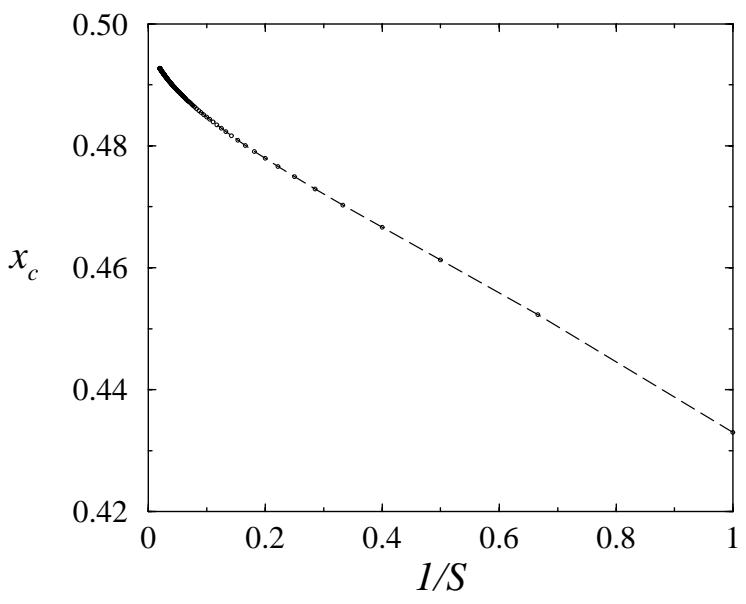

FIG. 1. Maximal concentration of holes $x_{c}(S)$ for the existence of a magnetization plateau vs $S^{-1}$ (the line is a guide to the eye only).

Tomonaga-Luttinger model. The analysis of the $T=0$ limit of the TBA Eqs. (2) shows that in phase $C$ only one of these modes is gapless [18]. The resulting low-energy theory is that of a single mode with dispersion,

$$
\epsilon_{2 S}(\xi)+\int_{-Q}^{Q} \mathrm{~d} \xi^{\prime} K\left(\xi-\xi^{\prime}\right) \epsilon_{2 S}\left(\xi^{\prime}\right)=\epsilon_{2 S}^{(0)}(\xi),
$$

where $K(\xi)=2 \sum_{k=1}^{2 S-1} a_{2 k}(\xi), \epsilon_{2 S}^{(0)}(\xi)=\left(2 S-\frac{1}{2}\right) H-$ $\mu-2 \pi \sum_{k=1}^{2 S} a_{2 k-1}(\xi)$, and $Q$ is a function of magnetic field and chemical potential through the condition $\epsilon_{2 S}( \pm Q)=0$. The corresponding hole concentration $x=\int_{-Q}^{Q} \mathrm{~d} \xi \sigma_{2 S}(\xi)$ is obtained from an equation for $\sigma_{2 S}$ similar to (4) with the driving term replaced by $\sigma_{2 S}^{(0)}(\xi)=\sum_{k=1}^{2 S} a_{2 k-1}(\xi)$. Further analysis of the zero temperature limit of the TBA Eqs. (2) shows that the massless mode in this phase carries the charge degrees of freedon while all magnetic excitations are gapped, i.e., $\kappa(\xi)<0$ and $\epsilon_{n \neq 2 S}(\xi)>0$ for all $\xi$. Hence, $x$ and the magnetization $M_{p}=S-3 x / 2$ are constant throughout this phase for fixed $Q$, i.e., $\left(2 S-\frac{1}{2}\right) H-\mu=$ const. This implies plateaus in the magnetization curve $M(H)$ below the saturated value $M_{s}=S-x / 2$ (see Fig. 2 for $S=1$ ). The end points of these plateaus are $H_{c 1}=-2 \mu$ and

$$
H_{c 2}=2 \mu+\frac{4}{S}+2 \int_{-Q}^{Q} \mathrm{~d} \xi a_{2 S-1}(\xi) \epsilon_{2 S}(\xi) .
$$

As $H \longrightarrow H_{c 1,2}$ from inside the plateau region, the spin gap closes as $\Delta \propto\left|H-H_{c 1,2}\right|$.

For finite temperatures the full set of TBA equations has to be solved to determine the magnetization curves. In a sufficiently strong magnetic field $H \gg T$, however, the energies $\epsilon_{n>2 S}$ are gapped and can be eliminated from the TBA Eqs. (2) [17]. For the doped $S=1$ chain this procedure leads to a coupled set of three nonlinear integral equations which are straightforward to solve by iteration. Choosing the chemical potential such that the hole 


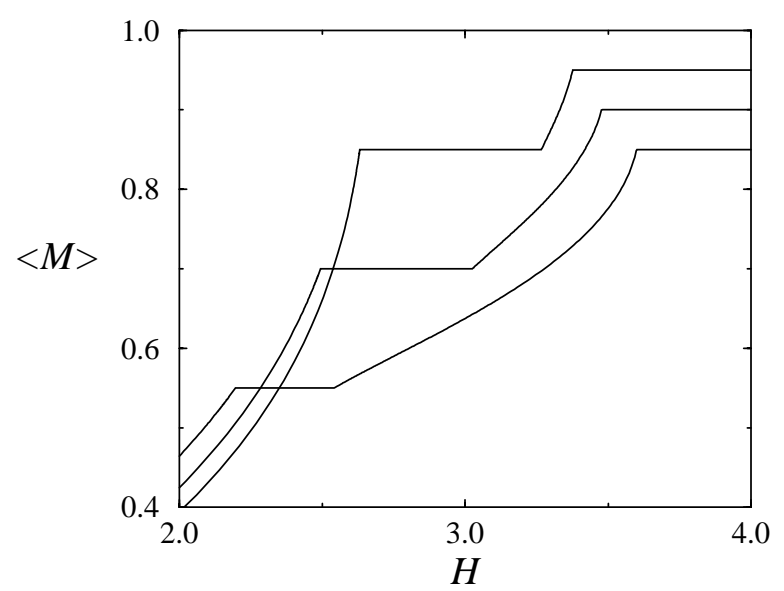

FIG. 2. Zero-temperature magnetization curve of the doped $S=1$ chain for the hole concentrations $x=0.1,0.2$, and 0.3 (top to bottom).

concentration $x=-\partial F / \partial \mu$ is fixed, the magnetization and magnetic susceptibility can be obtained from the thermodynamical potential $\Omega(T, H, x)=F(T, H, \mu)+\mu x$. In Fig. 3 we present the resulting data for various temperatures. They clearly show the formation of plateaus where the magnetic susceptibility becomes exponentially small with decreasing temperature as a consequence of the spin gap. In the same region, the specific heat is found to be $C=\pi T / 3 v_{2 S}$, i.e., linear in $T$, due to the presence of the gapless charge mode with Fermi velocity $v_{2 S}=\epsilon_{2 S}^{\prime}(Q) / 2 \pi \sigma_{2 S}(Q)$. In the vicinity of the transitions from the spin gap phase into the neighboring phases with Tomonaga-Luttinger liquid behavior, the singular behavior of the magnetization and susceptibility expected from the analysis of the zero-temperature phase diagram shows up.

Remarkably, the nature of these singularities on the two critical end points $H_{c 1,2}$ of the plateau is quite different: for the magnetic insulators discussed in the introduction the singular part of the magnetization near the plateaus has been predicted to show a square root behavior [1] due to the similarity of the transition to a commensurate-incommensurate transition [26]. A reliable numerical verification of this prediction-even for an integrable model - is extremely difficult for transitions other than the one into the ferromagnetically polarized state [7]. In the model considered here such difficulties arise for $H \rightarrow H_{c 1}$ only: for $H \leqslant H_{c 1}$ the zero temperature magnetization shows a critical behavior $\propto\left(H_{c 1}-H^{\alpha}\right)$ consistent with the square root behavior $\alpha=1 / 2$ within the numerical accuracy of our data. On the other hand, near $H_{c 2}$ the magnetization depends linearly on the external field, i.e., $M-M_{p} \propto H-H_{c 2}$ for $H \geqq H_{c 2}$. This difference in the critical behavior is also evident in the temperature dependence of the magnetic susceptibility near $H_{c 1,2}$, in particular, $\chi \approx$ const at the high-field end of the plateau [see Fig. 3(b)]. Note that a

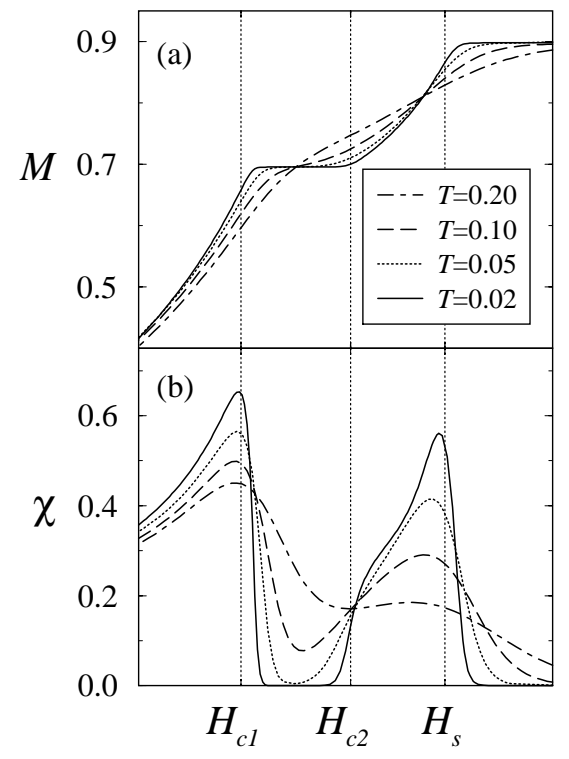

FIG. 3. Magnetization curve (a) and magnetic susceptibility (b) of the doped $S=1$ chain with hole concentration $x=0.2$ at temperatures $T=0.02,0.05,0.1$, and 0.2 [in units of (1)].

similar $T$ dependence has been observed in experiments on certain spin-1/2 Heisenberg ladders [27,28].

This different singular behavior is a consequence of the coupling between the two massless excitations present in the Tomonaga-Luttinger phases outside the interval $H_{c 1}<H<H_{c 2}$ : without the magnetic field breaking the spin-SU(2) these modes can be assigned usually to spin and charge excitations separately based on their different symmetries. In an external field, however, this assignment leads to a coupling of the two sectors. In certain cases this interaction can be removed by allowing for mixing of the corresponding quantum numbers [29]. Analyzing the Bethe ansatz wave functions we can determine the relation between the total charges $Q_{1,2}$ in the two gapless sectors to the physical quantum numbers, i.e., number of holes and $z$ component of the total spin. In the phase for $H<H_{c 1}$ a change in magnetization at fixed doping affects the total charge in one of the sectors only. Neglecting the coupling to the other sector, bosonization then gives the familiar square root singularity of the magnetization at $H \lesssim$ $H_{c 1}$ [26]. For $H>H_{c 2}$, however, a change of magnetization requires $\Delta Q_{1}=-\Delta Q_{2}$ for fixed doping $x$. Consequently, both the magnetic and the charge mode determine the critical behavior of the system as the critical field $H_{c 2}$ is approached from above. For $H \geqslant H_{c 2}$ the dispersion of the "incommensurate" soft mode can be approximated by the expression $\epsilon_{1}(k) \approx\left(v_{F}^{2} k^{2} / 4 \Delta\right)-\Delta$, where $\Delta \rightarrow$ $0^{+}$at the transition. From the analysis of the TBA equations, the field dependence of $\Delta$ is obtained as $\Delta \propto(H-$ $\left.H_{c 2}\right)^{2}$. This should be contrasted to the usual behavior $\Delta \propto\left|H-H_{c 2}\right|$ found in a system of free fermions. The quadratic field dependence immediately implies the linear $H$ dependence of the magnetization for $H \gtrsim H_{c 2}$. 
In summary, we have studied the properties of doped Heisenberg chains in a magnetic field in the framework of a Bethe ansatz solvable model. We find plateaus in the magnetization curves at certain values $M_{p}$ of the magnetization. To our knowledge this is a novel feature in an integrable model thereby providing the basis for more detailed studies aiming at a better understanding of the mechanism for the occurrence of these plateaus and the critical behavior at their end points. $M_{p}$ can be continuously tuned by changing the concentration of carriers. This is fundamentally different from the fixed values of $M_{p}$ obtained from topological arguments in the magnetic insulators studied previously. An extension of these arguments to the case of doped chains might be possible within a classical treatment of the localized $t_{2 g}$ spins in the double-exchange model [30]: in this limit the ground state has an incommensurate magnetic structure with $k_{F}$ periodicity [31]. However, unlike the models considered here this approach also leads to gapped charge excitations. An alternative attempt to describe the plateaus at tunable fractions of the maximal magnetization within a bosonized model relies just on this existence of a gapless charge mode [32]. Similarly, the second feature where the plateaus discussed here differ from the ones in quantum spin chains and ladders can be understood only as a consequence of the existence of a second massless mode: the relation of the physical quantum numbers to the conserved charges of the effective low-energy theory together with the coupling of the gapless channels conspire to give the linear field dependence of the singular part of the magnetization observed near the critical point $H_{c 2}$. Note that this mechanism does not restrict the critical exponents to the values $1 / 2$ and 1 discussed in this Letter.

Further studies of the low-energy properties - in particular, the analysis of the asymptotics of correlation functions as $H_{c 1,2}$ are approached-in this solvable microscopic model will lead to new insights into the critical behavior at the plateau transitions and possibly the related ones into Mott insulating phases of interacting particles. Furthermore, the phenomena reported in this Letter may be verified in experimental studies of the magnetization process in the doped, effectively one-dimensional transition metal oxides mentioned above.

We thank D.C. Cabra, F.H.L. Eßler, and A.M. Tsvelik for discussions. This work is supported in part by the Deutsche Forschungsgemeinschaft under Grant No. Fr $737 / 2$.

[1] M. Oshikawa, M. Yamanaka, and I. Affleck, Phys. Rev. Lett. 78, 1984 (1997).

[2] K. Hida, J. Phys. Soc. Jpn. 63, 2359 (1994).

[3] T. Tonegawa, T. Nakao, and M. Kaburagi, J. Phys. Soc. Jpn. 65, 3317 (1996).
[4] K. Okamoto, Solid State Commun. 98, 245 (1996).

[5] K. Totsuka, Phys. Rev. B 57, 3454 (1998).

[6] D. C. Cabra, A. Honecker, and P. Pujol, Phys. Rev. Lett. 79, 5126 (1997).

[7] D. C. Cabra, A. Honecker, and P. Pujol, Phys. Rev. B 58, 6241 (1998).

[8] T. Sakai and M. Takahashi, Phys. Rev. B 57, R3201 (1998).

[9] R. Sato and K. Kindo, Physica (Amsterdam) 246B-247B, 372 (1998).

[10] A. Fledderjohann, C. Gerhardt, M. Karbach, K.-H. Mütter, and R. Wiessner, Phys. Rev. B 59, 991 (1999).

[11] T. Sakai and Y. Hasegawa, Phys. Rev. B 60, 48 (1999).

[12] T. Momoi, H. Sakamoto, and K. Kubo, Phys. Rev. B 59, 9491 (1999).

[13] W. Chen, K. Hida, and H. Nakano, J. Phys. Soc. Jpn. 68, 625 (1999).

[14] Y. Narumi et al., Physica (Amsterdam) 246B-247B, 509 (1998).

[15] H. Tanaka et al., Physica (Amsterdam) 246B-247B, 230 (1998).

[16] W. Shiramura et al., J. Phys. Soc. Jpn. 67, 1548 (1998).

[17] H. Frahm, M. P. Pfannmüller, and A. M. Tsvelik, Phys. Rev. Lett. 81, 2116 (1998).

[18] H. Frahm, Nucl. Phys. B559, 613 (1999).

[19] C. Zener, Phys. Rev. 82, 403 (1951); P. W. Anderson and H. Hasegawa, Phys. Rev. 100, 675 (1955).

[20] E. Dagotto, J. Riera, A. Sandvik, and A. Moreo, Phys. Rev. Lett. 76, 1731 (1996).

[21] E. Müller-Hartmann and E. Dagotto, Phys. Rev. B 54, R6819 (1996).

[22] F. C. Zhang and T. M. Rice, Phys. Rev. B 37, 3759 (1988).

[23] E. Dagotto et al., Phys. Rev. B 58, 6414 (1998).

[24] L. Takhtajan, Phys. Lett. 87A, 479 (1982); H. M. Babujian, Phys. Lett. 90A, 479 (1982).

[25] H. M. Babujian, Nucl. Phys. B215, 317 (1983).

[26] G. I. Japaridze and A. A. Nersesyan, JETP Lett. 27, 334 (1978); H. J. Schulz, Phys. Rev. B 22, 5274 (1980).

[27] G. Chaboussant et al., Phys. Rev. B 55, 3046 (1997).

[28] W. Shiramura et al., J. Phys. Soc. Jpn. 66, 1900 (1997).

[29] This situation is well known from the analysis of correlation functions of the one-dimensional Hubbard model in a magnetic field where the conformal radius of the bosonic mode associated with the charge degrees of freedom varies continuously with $H$ while the low-lying magnetic excitations evolve from spinons carrying spin $1 / 2$ for vanishing field to magnons with spin 1 as $H \rightarrow H_{s}$ [see H. Frahm and V.E. Korepin, Phys. Rev. B 43, 5653 (1991)].

[30] Note that the Hamiltonian of this model will have little in common with the $S \rightarrow \infty$ limit of the integrable models considered here which will not be a polynomial in $\mathbf{S}_{i} \cdot \mathbf{S}_{j}$; see, e.g., L. D. Faddeev, in Quantum Symmetries, Proceedings of the Les Houches Summer School, Session LXIV, edited by A. Connes, K. Gawedzki, and J. Zinn-Justin (Elsevier, Amsterdam, 1998).

[31] W. Koshibae, M. Yamanaka, M. Oshikawa, and S. Maekawa, Phys. Rev. Lett. 82, 2119 (1999).

[32] D.C. Cabra, A. De Martino, A. Honecker, P. Pujol, and P. Simon, cond-mat/9908398. 